$$
-7
$$

NASA Technical Memorandum 107605

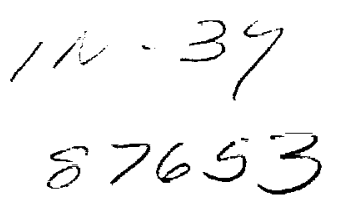

p.12

\title{
COLLAPSE OF COMPOSITE TUBES UNDER END MOMENTS
}

Alan E. Stockwell and Paul A. Cooper

April 1992

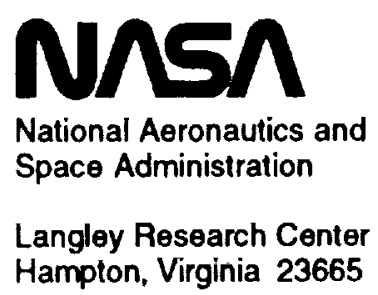

(NASA-TM-107605) COLLAPSE OF COMPOSITE

N92-23992

TUBES

UNDER END MOMENTS

(NASA)

$12 \mathrm{P}$ 


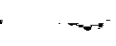

-

r 


\title{
COLLAPSE OF COMPOSITE TUBES UNDER END MOMENTS
}

\author{
Alan E. Stockwell * \\ Lockheed Englneering and Sciences Company \\ Hampton, Virginia \\ and \\ Paul A. Cooper "* \\ NASA Langley Research Center \\ Hampton, Virginia
}

\begin{abstract}
Cylindrical tubes of moderate wall thickness such as those proposed for the original space station truss, may fail due to the gradual collapse of the tube cross-section as it distorts under load. Sometimes ieferred to as the Brazler instability, it is a nonlinear phenomenon. This paper presents an extension of an approximate closed-form solution of the collapse of isotropic tubes subject to end moments developed by Reissner in 1959 to include specially orthotropic material. The closed-form solution was verified by an exiensive nonlinear finite element analysis of the collapse of long tubes under applied end moments for radius-to-thickness ratios and composite layups in the range proposed for recent space station truss framework designs. The finite element analysis validated the assumption of inextensional deformation of the cylindrical cross-section and the approximation of the material as specially orthotropic.
\end{abstract}

\section{Introduction}

Designers of large truss-type space structures, including designers of the original space station configuration, proposed using composite tubes as basic truss elements. The tubes were primarily designed to resist Euler buckling. However, manned structures like the space station also have to support bending moments caused by an astronaut pushing against a tube or caused by other transverse forces which might occur during the normal operation of transportation platforms. In these situations, a thinwalled tube subjected to a bending moment can fail in a short wave-length instability mode due to excessive local compressive stress, whereas a thickwalled tube can tail due to stresses in excess of the ultimate strength of the material. For tubes of moderate wall thickness, such as those proposed for the original space station truss, another mode of failure can occur. The failure is characterized by the gradual collapse of the tube because of reductions in

- Supervisor, Struclural Dynamics Section, member AIAA, ASCE

* Senior Research Engineer, Spacecraft Dynamics Branch, associate fellow AIAA, member ASME its moment of inertia due to cross-sectional distortion under load. This failure mode is generally referred to as Brazier instability, and is a nonlinear phenomenon.

The nonlinear problem of pure bending of infinitely long straight tubes was first solved by Brazier. 2 Wood ${ }^{3}$ developed the theory further to include the effects of pressure, and Reissner ${ }^{1}$ refined the solution and expanded it to include initially curved tubes. Axelrad and Emmerling ${ }^{4}$ provided a numerical solution of the local instability about the deformed state of a short finite-length, orthotropic tube under bending. The present paper extends an approximate closed-form solution of the collapse of long isotropic tubes subject to end moments developed by Reissner in 1959 to include a special class of tubes made of laminated composite material. The theory presented herein is developed for unpressurized straight tubes, and requires that the composite behaves as a specially orthotropic homogeneous material in a state of plane stress. Therefore fiber orientations and stacking sequence of a laminate modeled by this theory must yield physical properties which are consistent with this assumption. These physical requirements and their effects on the constitutive equations for a general multi-layered composite material are discussed in the paper. The closed-form solution is verified by an extensive nonlinear finite element analysis of the collapse of long tubes under applied end moments for radius-tothickness ratios and composite layups in the range proposed for recent space station truss framework designs.

\section{Extension of Closed-form Solution to Composite Tubes}

The analysis in this section extends the nonlinear collapse analysis presented by Reissner for isotropic tubes to include tubes made of specially orthotropic composite materials. The extension of the analysis requires only minor modifications to Reissner's theory. For completeness, an overview of the theory and the corresponding modifications are presented in this section. Specifically, the basic assumptions of Reissner's theory are presented and the implications of the assumptions on composite tube construction are discussed. Next, the modifications to specific parts of Reissner's theory are presented and the equations governing the collapse behavior of composite tubes are derived. 


\section{Assumotions of Reissner's Theory}

An exact analysis for predicting the nonlinear collapse of tubes subjected to pure bending moments is difficult to obtain in closed form. However, Relssner introduced some simplifying assumptions that permit formulation of an approximate closed-form solution. The assumptions simplify the analysis by capturing only the dominant mechanisms participating in the actual collapse response.

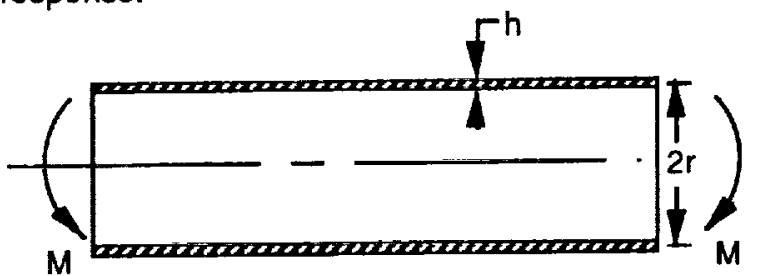

Figure 1 Longitudinal bending of an initially straight tube

A tube is considered which has a wall thickness $h$, mean cross-sectional radius $r$, and is subjected to a moment $M$ applied to the ends of the lube, as shown in figure 1. As the tube bends, axial stresses develop in the shell wall. In Reissner's theory, these stresses and the corresponding strains are assumed to be primarily due to the beam bending action of the tube and are assumed to be uniform along the length of the tube. Local bending of the shell is assumed to be negligible. However, the orientation of the longitudinal stresses induces stresses in the plane of the tube cross-section which cause the cross-section to deform in a doubly symmetric manner as shown in figure 2. As the tube

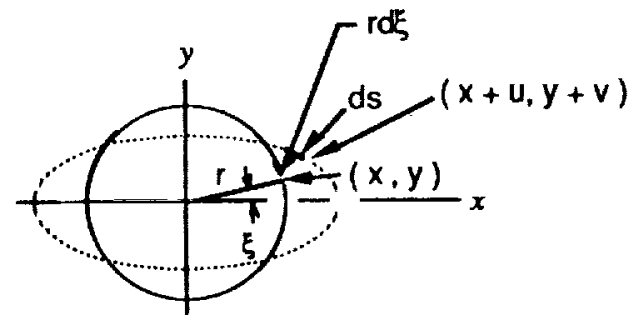

Figure 2 Cross-sectional deformation

cross section deforms, stresses and strains develop within the cross-section. The cross-sectional deformation state is assumed to be identical for all cross-sections. An important assumption made by Reissner which greatly simplifies the analysis is that each cross-section remains inextensible during deformation, i.e., the circumferential membrane strain is negligible compared to the corresponding bending strain caused by changes in the profile. The mathematical implications of these assumptions are presented in subsequent sections of the present study.

\section{Bestrictions on Cemposite Tube Construction}

The equations relating the stress resultants to the membrane strains and curvature changes of a general multi-layered laminate under plane stress $\operatorname{are}^{5}$

$$
\begin{gathered}
\left\{\begin{array}{c}
N_{p} \\
N_{q} \\
N_{p q}
\end{array}\right\}=\left[\begin{array}{lll}
A_{11} & A_{12} & A_{16} \\
A_{12} & A_{22} & A_{26} \\
A_{16} & A_{26} & A_{66}
\end{array}\right]\left\{\begin{array}{l}
\varepsilon_{p}^{0} \\
\varepsilon_{q}^{0} \\
\gamma_{p q}^{0}
\end{array}\right\} \\
+\left[\begin{array}{lll}
B_{11} & B_{12} & B_{16} \\
B_{12} & B_{22} & B_{26} \\
B_{16} & B_{26} & B_{66}
\end{array}\right]\left\{\begin{array}{c}
\kappa_{p}^{0} \\
\kappa_{q}^{0} \\
\kappa_{p q}^{0}
\end{array}\right\}
\end{gathered}
$$

and

$$
\begin{aligned}
& \left.\mid \begin{array}{c}
M_{p} \\
M_{q} \\
M_{p q}
\end{array}\right\}=\left[\begin{array}{lll}
B_{11} & B_{12} & B_{16} \\
B_{12} & B_{22} & B_{26} \\
B_{16} & B_{26} & B_{66}
\end{array}\right]\left(\begin{array}{c}
\varepsilon_{p}^{0} \\
\varepsilon_{q}^{0} \\
\gamma_{p q}^{0}
\end{array}\right\} \\
& +\left[\begin{array}{lll}
D_{11} & D_{12} & D_{16} \\
D_{12} & D_{22} & D_{26} \\
D_{16} & D_{26} & D_{66}
\end{array}\right]\left\{\begin{array}{c}
\kappa_{p}^{0} \\
\kappa_{q}^{0} \\
\kappa_{p q}^{0}
\end{array}\right\}
\end{aligned}
$$

where $N_{p}, N_{q}$, and $N_{p q}$ are the in-plane distributed forces; $M_{p}, M_{q}$, and $M_{p q}$ are the distributed bending and twisting moments; $\varepsilon_{p}{ }^{0}, \varepsilon_{q}{ }^{0}$, and $\gamma_{p q}{ }^{0}$ are the extensional and shear strains of the middle surface; and $\kappa_{p}{ }^{0}, \kappa_{q}{ }^{0}$, and $\kappa_{p q}{ }^{0}$ are the changes in curvature of the middle surface. The subscripts $p$ and $q$ refer to an arbitrary body coordinate system. The elements of the A, B, and D matrices are in general non-zero; however, equations (1) and (2) may be reduced to the equations for a homogeneous orthotropic material if the stacking sequence and fiber orientation of the plies has a certain arrangement.

The class of laminates investigated in the present study have geometry and material properties which are symmetric with respect to the middle surface of the shell. Therefore there is no bendingextension coupling and the $B_{i j}$ terms in equations (1) and (2) are all zero. The $A_{16}$ and $A_{26}$ terms in equation (1) are also zero for these laminates, i.e., there is no shear-extension coupling, because the material layups for the tubes considered in the present study have a lamina oriented at $-n$ degrees to cancel the effect of each lamina oriented at $+n$ degrees. An assumption is made that the $D_{16}$ and $D_{26}$ terms in equation (2) are zero, i.e., there is no bending-iwisting coupling. Although this is not generally true, there are many practical laminates in which the effects of these terms are negligible. The assumptions lead to the result that the principal material axes, 1 and 2, of the laminate are aligned with the natural body axes, $p$ and $q$, and the material is referred to as specially orthotropic. Under these conditions, equations (1) and (2) reduce to 


$$
\begin{aligned}
& \left\{\begin{array}{c}
N_{1} \\
N_{2} \\
N_{12}
\end{array}\right\}=\left[\begin{array}{ccc}
A_{11} & A_{12} & 0 \\
A_{12} & A_{22} & 0 \\
0 & 0 & A_{66}
\end{array}\right]\left(\begin{array}{c}
\varepsilon_{1}^{0} \\
\varepsilon_{2}^{0} \\
\gamma_{12}^{0}
\end{array}\right\} \\
& \left\{\begin{array}{c}
M_{1} \\
M_{2} \\
M_{12}
\end{array}\right\}=\left[\begin{array}{ccc}
D_{11} & D_{12} & 0 \\
D_{12} & D_{22} & 0 \\
0 & 0 & D_{66}
\end{array}\right]\left(\begin{array}{c}
\kappa_{1}^{0} \\
\kappa_{2}^{0} \\
\kappa_{12}^{0}
\end{array}\right\}
\end{aligned}
$$

In the following discussion, the principal material directions, 1 and 2, are aligned with the longitudinal and circumferential directions of the tube, respectively. For example, the longitudinal stress in the tube is denoted by $\sigma_{l}$.

\section{Beam Bending Effects}

The equilibrium equation for beam bending, relating the applied moment to the longitudinal fiber stress $\sigma_{l}$, is given by

$$
M=\int h \sigma_{1}(y+v) d s
$$

Where $d s$ and $y+v$ are defined as shown in figure 2. From equation (3), the relation between the fiber stress $\sigma_{l}$ and the fiber strain at the midsection of the tube wall $\varepsilon_{l}{ }^{0}$ is

$$
\sigma_{1}=\frac{1}{h}\left(A_{11} \varepsilon_{1}^{0}+A_{12} \varepsilon_{2}^{0}\right)
$$

Due to Reissner's assumption that the circumferential strain of the middle surface of the tube wall is negligible, the equation simplifies to

$$
\sigma_{1}=\frac{\Lambda_{11}}{h} \varepsilon_{1}^{0}
$$

The term $\sigma$, represents an average beam-like stress, and since it is constant along the length of the beam and is uniquely related to the applied moment, it can be treated as an applied stress in the problem formulation. The cross-sectional strain distribution, with the influence of the cross-sectional deformation, is expressed as

$$
\varepsilon_{1}^{0}=\frac{(y+v)}{R}
$$

\section{Cross-sectional Deformation Effects}

In Reissner's simplified nonlinear analysis, it is assumed that the deformation of the tube crosssection is caused primarily by loading induced into the plane of the tube cross-section by the longitudinal beam bending stress $\sigma_{1}$. As the tube bends, the longitudinal stress follows the deformed geometry, and a force is projected into the plane of the crosssection. The resulting force is then transformed into an equivalent pressure $p_{\sigma}$ that acts along the $y$ axis as shown in figure 3.

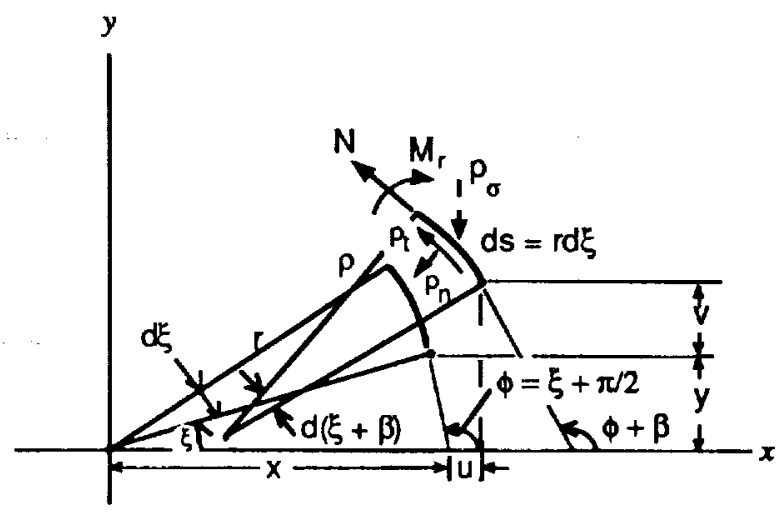

Figure 3 Change in curvature and tangent angle, and forces acting on cross-sectional segment

The pressure is given by

$$
P_{\sigma}=\sigma_{1} \frac{h}{R}
$$

and the components in the normal and tangential directions are

$$
\begin{aligned}
& p_{n}=p_{\sigma} \sin (\xi+\beta) \\
& p_{1}=-p_{\sigma} \cos (\xi+\beta)
\end{aligned}
$$

Figure 3 shows the resulting deformation of a differential segment of the cylinder's cross-section. The original tangent angle of the segment changes from $\phi$ to $\phi+\beta$, and $\beta$ is taken as the deformation variable. The deformed radius of curvature expressed as a function of $\beta$ is

$$
\frac{1}{\rho}=\frac{1}{r}\left(1+\beta^{\prime}\right)
$$

where $r$ is the undeformed tube radius and $\rho$ is the radius of the deformed differential segment. Primed quantities are differentiated with respect to $\xi$. The displacement $v$ in the $y$ direction is found to be

$$
v=r \int_{0}^{\xi}[\cos (\psi+\beta)-\cos \psi] d \psi
$$

where $\xi$ is the angular coordinate of the middle surface of the cross-section, and $\psi$ is a dummy integration variable.

The forces acting on the deformed crosssection are also shown in figure 3 , and the corresponding equilibrium equations are

$$
\underline{N^{\prime}} \cdot \frac{Q}{\rho}+p_{t}=0
$$




$$
\begin{aligned}
& \frac{Q^{\prime}}{r}+\frac{N}{\rho}+p_{n}=0 \\
& \frac{M_{r}^{\prime}}{r}-Q=0
\end{aligned}
$$

where $Q$ is the shear force per unit length, and $p_{t}$ and $p_{n}$ are the tangential and normal components of pressure.

From equation (4), the circumferential moment is given in terms of the change of curvature as

$$
M_{r}=M_{2}=D_{12} k_{1}^{0}+D_{22} \kappa_{2}^{0}
$$

Consistent with Reissner's formulation for isotropic materials, the $D_{12}$ term in equation (17) is ignored, i.e., the longitudinal curvature is assumed to be much smaller than the curvature caused by the crosssectional bending. For the tubes used in this study, it can be demonstrated that this assumption is reasonable, i.e., $\kappa_{l}{ }^{0}$ is on the order of one percent of $\kappa_{2}{ }^{\circ}$ at collapse. The twisting curvature $\kappa_{12}{ }^{0}$ should also be zero due to the initial assumption of uniform bending (constant curvature). In other words, the assumption that $D_{16}$ is zero should not cause appreciable errors unless $D_{16}$ is very large. Therelore equation (17) reduces to

$$
M_{r}=D_{22} \kappa_{2}^{0}
$$

and the stress-strain relation becomes

$$
\frac{M_{L}}{D_{22}}=\kappa_{2}^{\Omega}=\frac{1}{r}-\frac{1}{\rho}=-\frac{\beta}{r}
$$

\section{Equations Governing Collapse Behavier and} Analytical Solution

The system of equations (9) to (11) and (14) to (19) is further reduced. Trigonometric terms with $\beta$ in the argument are expanded and terms of order $\beta^{3}$ are neglected. Symmetric boundary conditions are applied, and with appropriate substitutions, the modified form of the final nonlinear ordinary differential equation for the deformation variable $\beta$ is found to be

$$
\begin{aligned}
& \frac{D_{22}}{r^{3}}\left\{\beta^{i v}+\beta^{\prime \prime}-\beta^{\prime}\left[\beta^{i v}-\beta^{\prime \prime}\right]-\beta^{\prime \prime} \beta^{\prime \prime \prime}\right\} \\
& =\frac{r A_{11}}{R^{2}}\left\{\frac{3}{2} \sin 2 \xi-2 \cos \xi \int_{0}^{\xi} \beta \sin \psi d \psi\right. \\
& \left.\quad+\left(\cos ^{2} \xi-3 \sin ^{2} \xi\right) \beta-\frac{1}{2} \beta^{\prime} \sin 2 \xi-\beta^{\prime \prime} \sin ^{2} \xi\right\}
\end{aligned}
$$

The equation for the fiber stress $\sigma_{l}$ becomes

$$
\frac{\sigma_{1} h}{\Lambda_{11}}=\frac{r}{R}\left\{\sin \xi-\int_{0}^{l}\left(\beta \sin \psi+\frac{1}{2} \beta^{2} \cos \psi\right) d \psi\right\}
$$

and the moment-curvature equation becomes

$$
\begin{aligned}
\frac{M h}{A_{11} I}= & \frac{1}{R}\left\{1-\frac{2}{\pi} \int_{0}^{2 \pi}\left[\int_{0}^{\xi} \beta \sin \psi d \psi\right] \sin \xi d \xi\right. \\
& -\frac{1}{\pi} \int_{0}^{2 \pi}\left[\left(\int_{0}^{\xi} \beta^{2} \cos \psi d \psi\right) \sin \xi\right. \\
& \left.\left.-\left(\int_{0}^{\xi} \beta \sin \psi d \psi\right)^{2}\right] d \xi\right\}
\end{aligned}
$$

Reissner's method of solution for (20), (21) and (22) is equally valid for the modified equations, because only the constant coefficients of the differential equations have been altered. The equation for $\beta$, equation 20 , is solved by expanding $\beta$ in powers of a parameter $\alpha^{2}$, where $\alpha^{2}$ is expressed as

$$
\alpha^{2}=\frac{r^{4} A_{11}}{8 D_{22} R^{2}}
$$

Retaining only the first two terms of the expansion, consistent with the prior assumption of dropping terms of order $\beta^{3}$, the approximate solution for the moment-curvalure change equation is

$$
m=\alpha^{2}\left[1-\alpha^{2}-\frac{2}{3}\left(\alpha^{2}\right)^{2}\right]
$$

where

$$
m=\frac{M h r^{2}}{A_{11} l}\left[\frac{A_{11}}{8 D_{22}}\right]^{1 / 2}
$$

and $l$ is the undeformed moment of inertia of the tube cross-section. Equations (24) and (25) relate the cross-sectional distortion to the applied moment. The tube collapses when the moment attains a maximum value, which is oblained by solving for the value of $\alpha^{2}$ which maximizes $m$ in equation (24). The critical value of $\alpha^{2}$ is 0.509 and the corresponding critical value of $m$ is

$$
m_{c}=0.354
$$

Substitution of (26) into (25) and substitution of $I=\pi r^{3} h$ as an approximation of the moment of inertia: yields the following equation for $M_{c r}$. the moment at which a moderately thick-walled tube subject to applied end moments collapses due to loss of crosssectional stiflness: 


$$
M_{c r}=\pi r\left[A_{11} D_{22}\right]^{1 / 2}
$$

Reissner shows that the amount of crosssectional flattening. $\delta_{c r}$, associated with $M_{c r}$ depends only on the critical value of $\alpha^{2}$ and would not be affected by the assumption of a specially orthotropic material. Therefore his computed value of $\delta_{c r}$ still applies, so that from reference 1 ,

$$
\delta_{c r}=0.207 r
$$

Note that $\delta_{c r}$ in the first approximation, depends only on the tube's original cross-sectional radius and is independent of the tube thickness $h$.

\section{Yylldatlon of Extended Closed-form Solution}

An efficient computational approach was desired to validate the extended closed-form solution for tubes having properties in the range of interest for this study. Nonlinear collapse analyses of isotropic tubes conducted in the past using computational methods include a study by Stephens, Starnes and Almroth6, who analyzed the nonlinear collapse behavior of long cylindrical shells subjected to combined bending and pressure as well as pure bending using a finite difference approach. Their results showed close agreement with the predictions of Brazier ${ }^{1}$ for isotropic tubes with a radius-tothickness $(r / t)$ ratio of 100 and loaded by pure bending. Knight, Macy, and McCleary ${ }^{7}$, using a finite element approach to analyze the collapse of long circular isotropic cylinders in bending. reported similar results. However, these analyses included neither orthotropic materials nor tubes with $r / t<100$.

The present study was motivated by the proposed use of graphite-epoxy tubes having $\mathrm{r} / \mathrm{t}$ ratios much less than 100 as structural elements in large space structures. A typical section, or bay, of such a structure is sketched in figure 4 . The structure

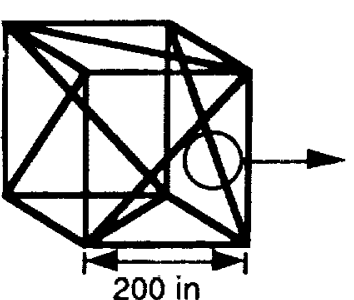

Typical Truss Bay

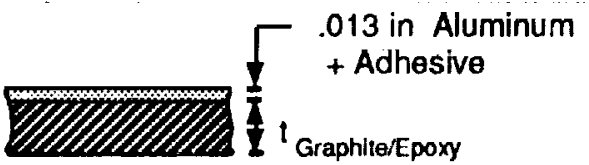

SECTION A-A

Figure 4 Proposed space structure tube properties is composed of a series of individual truss members connected in a repeating geometric pattern. Each truss member is a 2.5-inch diameter tube with a wall thickness of 0.0784 inches plus a 0.013 inch thick non-structural protective aluminium coating bonded to the graphite-epoxy. Table 1 lists the properties of the graphite-epoxy material which was used as a baseline for this study.

Tube Configuration:

Number of plies:

Ply thickness:

Layup:

16

0.0052 in.

$\left[28 / 0_{4} /-28 / 0_{2}\right]_{s}$

Coating:0.005 in. aluminum foil bonded by 0.008 in. adhesive layer

Material Design Properties for IM7/977-2:

(Fiber Volume and density: $V_{\mathbf{I}}=\mathbf{5 7 . 6 \%}$, $\left.\rho=0.056 \mathrm{lb} / \mathrm{in}^{3}\right)$

$\begin{array}{ll}E_{1} & =20 E 6 \text { psi } \\ E_{2} & =1.06 E 6 \text { psi } \\ G_{12} & =0.75 E 6 \text { psi } \\ v_{12} & =0.3\end{array}$

Lamina Strength Properties:

(Note: $X=$ fiber, $Y=$ matrix, $T=$ tension, $C=$ compression)

$X_{T}=320 \mathrm{E3} \mathrm{psi}$
$X_{C}=160 \mathrm{E} 3 \mathrm{psi}$
$Y_{T}=8.5 \mathrm{E} 3 \mathrm{psi}$
$Y_{C}=30 \mathrm{E} 3 \mathrm{psi}$

$S \quad=11 \mathrm{E} 3$ psi (in-plane shear strength) IS $=14 \mathrm{E} 3$ psi (interlaminar shear strength)

Table 1 Tube layup and lamina strength properties for baseline composite design

The following section describes the finite element model and analysis procedure used in the study. The accuracy of the analytical model was established by performing a finite element analysis of an isotropic tube and comparing the results to Reissner's ${ }^{1}$ solution. Orthotropic material properties were substituted into the model and the same analysis procedure was used to validate the extended Reissner solution for the laminate design which was chosen as a baseline for this study. Finite element analyses were then performed for a range of laminate designs to extend the validation of the closed form solution.

\section{Einite Element Medel}

A finite element model was constructed of a section of tube with the geometry shown in figure 5 . A quarter section of a tube of length $L$ was modeled using symmetric boundary conditions in the $x z$ and $y z$ planes, A moment $M$ was applied at one end of the tube using a "rigid element". The rigid element consisted of constraint equations which forced the 
circular cross-section to remain circular and rotate as a rigid body about the $y$ axis.

An initial finite element model was built for a tube with a length to radius ratio $(L / r)$ of 10 . The model, shown in figure 5 , consisted of 16 equally spaced rings of quadrilateral elements. Each ring contained 36 elements evenly spaced at 5 degrees apart. The four rings closest to the loaded edge were made slightly thicker than the nominal tube wall thickness. The thickness of the ring at the loaded edge was increased enough to provide a 10 percent increase in moment of inertia of the nominal tube cross-section and each of the other three rings was designed to provide 2.5 percent less inertia than the previous ring. The tapered wall thickness was introduced to prevent failure due to local instabilities at the loaded edge.

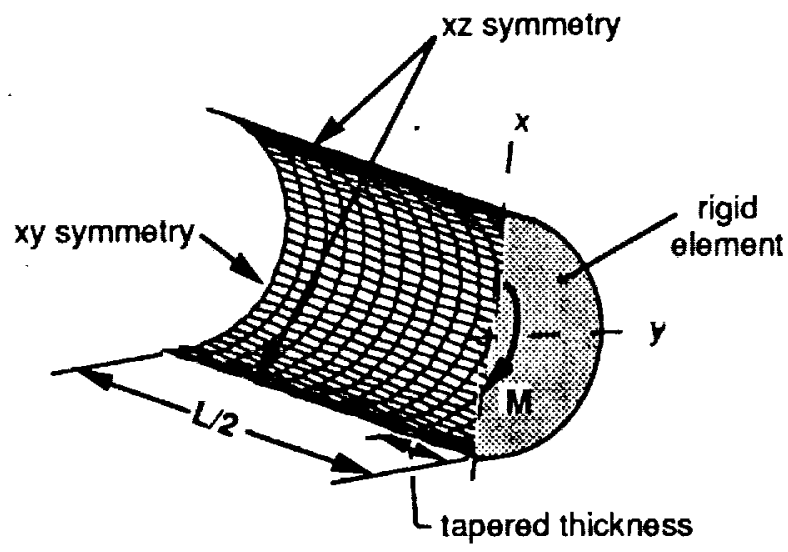

Figure 5 Finite element model for $L / r=10$

The model was analyzed using MSC/NASTRAN to solve the geometric nonlinear problem using an iterative process based on variations of Newton's method. 8 An analysis was selected which allowed the program to automatically choose the most efficient solution strategy and update the tangent stiffness matrix when necessary based on estimated convergence criteria. The moment was applied incrementally to the fube and the total amount of cross-sectional flattening was computed at each converged load step. Figure 6 illustrates that the cross-sectional flattening, $\delta_{w}$, is defined as the average of the absolute values of the elastic deformations of the top and bottom of the tube. It is measured at the unloaded end of the model and is calculated by taking half the absolute value of the difference of the transverse deflections at $\xi=\pi / 2$ and $\xi=-\pi / 2$. The collapse moment was defined as the moment at which a load increment of less than 0.1 percent caused the solution to fail to converge.

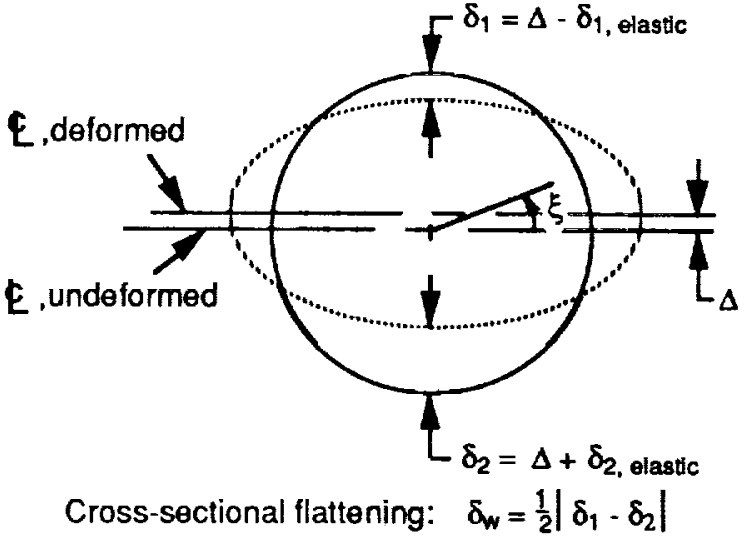

Note: maximum cross-sectional flattening occurs at $L / 2$, i.e., at the xy symmetry plane of the model

Figure 6 Definition of cross-sectional flattening

\section{Accuracy of the Finite Element Model}

The initial analyses conducted in the present study used tubes with isotropic materials in order to verify the modeling and solution procedures and to provide a lower bound for the collapse moment of the baseline composite tube. Therefore, material properties were chosen for an isotropic material having a Young's modulus equal to $E_{22}$ (the weaker, circumferential modulus) of the composite material. A length-to-radius ratio of 10 was chosen for the first model to minimize the number of finite elements needed in the analysis. An initial analysis was performed without the rigid element at the loaded end to allow the cross-section to deform and more closely approximate a pure bending loading through a larger region of the model. Moment loading was accomplished by applying an axial edge force distributed as a cosine function around the circumference of the tube. However failure caused by local instabilities occurred at the loaded edge when the applied moment reached a value of about 60 percent of the predicted collapse moment.

In order to prevent the premature edge failure, the rigid element, described previously, was added to the model to ensure that the loaded edge would not buckle. The revised model was loaded until collapse of the cross-section occurred at the end of the tube where the symmetric boundary conditions are applied. A plot of applied moment versus crosssectional flattening at the end of the tube, shown in figure 7. indicates that the final collapse moment, 9515 in-lbs, was 5.7 percent greater than the predicted value obtained by using Reissner's solution. The cross-sectional flattening, $\delta_{w}$, was 0.136 inches, which was 45 percent less than the value predicted by the Reissner solution. 


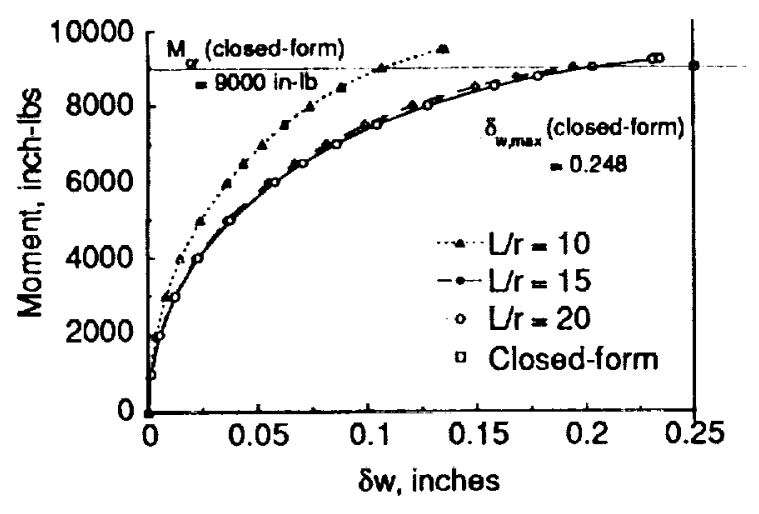

Figure $7 M$ vs $\delta_{w}$ for various ratios of $L r$, isotropic material, $E=E_{22}=1.29 E 6$ psi

\section{Length Effect}

Two additional models were analyzed to investigate the effect of tube length on the predicled collapse moment. One model was built to represent a tube with an $L / r$ ratio of 15 and one with an $L / r$ of 20. The results are plotted in figure 7 along with the results of the first analysis. The predicted collapse moment for the longest tube $(L / r=20)$ was $9220 \mathrm{in}-\mathrm{lb}$, which was 2.4 percent greater than the predicted moment, and the cross-sectional flattening was 5.6 percent less than the predicted value. The symbols on the curves in figure 7 , which indicate intermediate solution results, show that the convergence criteria also affected the cross-sectional flattening more than the collapse moment. References 6 and 7 show similar trends for isotropic cylinders with $R / t$ ratios of 100 and varying $L r$ ratios.

The length effect was further investigated by plotting the cross-sectional flattening as a function of the distance from the loaded edge of the tube, as shown in figure 8 . In the finite element models, the

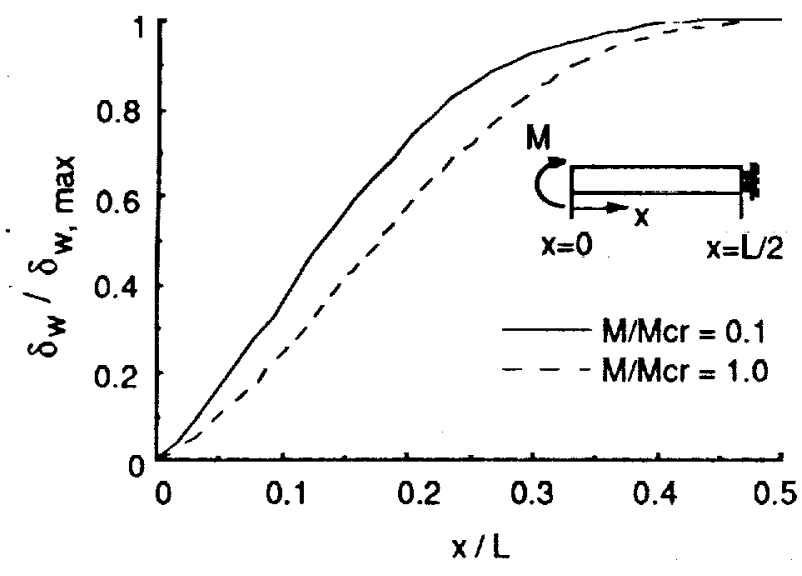

Figure 8 Normalized $\delta_{w}$ vs $x / L$ for isotropic material, $\mathrm{L} / \mathrm{r}=20$

rigid element used to load the beam introduced boundary conditions which affected the deformation. causing the maximum $\delta$, to occur at a distance of $L / 2$ from the loaded end ( $L / 2$ is the actual length of the model, since it is a symmetric representation of a tube of length $L$ ). However, according to Reissner's theory, $\delta_{w}$ should be constant along the length of the beam, since the beam is infinitely long and is in a state of pure bending. The plot in figure 8 shows that although the cross-sectional deflection varied from zero at the loaded end to $\delta_{w, \max }$ at $x=L / 2$, the deformation in the region $0.4 L$ to $0.5 L$ was relatively constant and was within 95 percent of the maximum. Based on the results shown in figures 7 and 8 , it was concluded that an $L / r$ of 20 was sufficient to approximate pure bending at $L / 2$, and a decision was made to use this $L / r$ ratio for the rest of the models in the present study.

\section{Nonlinear Bending of Elastic Axis}

The nonlinear character of the bending is further illustrated in figure 9, where a plot is shown of the applied moment versus the deflection of the elastic axis of the tube for the $L / r=20$ case. A comparison to the straight-line deflection curve predicted by linear beam-bending theory shows that the cross-sectional deformation causes a significant reduction in stiffness at load levels well below the collapse moment. The actual deflection of the elastic axis at collapse is about 30 percent greater than the deflection which is predicted by linear beam theory for the same applied moment.

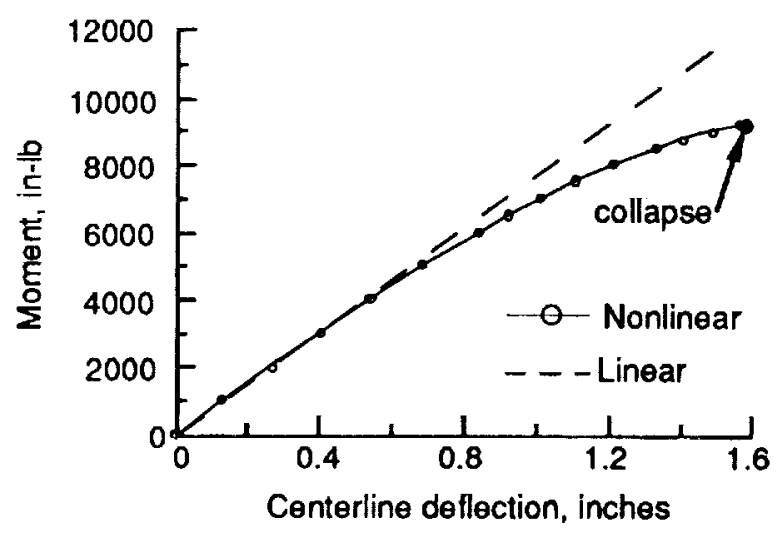

Figure 9 Deflection of elastic axis, isotropic material

\section{Besults}

The finite element solution agreed with the Reissner solution for the critical collapse moment of isotropic tubes with an $L / r$ ratio of about 20 by predicting a critical moment which was less than 3 percent different. The cross-sectional deformation was relatively constant in the central portion of the tube, indicating that the finite element model provided a good approximation to pure bending of an infinitely long tube at the collapse location. Even though both the critical moment and the maximum cross-sectional flattening were functions of the model length, the cross-sectional flattening was much more sensitive to both the model length and the definitior. of the convergence criteria for the collapse moment. 
Inspection of the deformed cross-section prior to collapse revealed that the deformation was not doubly symmetric, as assumed in the closed form solution.

\section{Analysis of Composite Tubes}

The verified isotropic finite element model was modified slightly for the analysis of composite tubes. The lamina properties and layup of the composites were used as input to the finite element program, which calculated equivalent orthotropic properties to use in the nonlinear finite element analyses. The calculated properties included the $D_{16}$ and $D_{26}$ terms, which were ignored in the closed-form solution. The same analysis procedure used in analyzing the isotropic tubes was followed.

\section{Baseline Composite}

The material properties and layup listed in table 1 were provided as input for analyzing the composite tube used as a baseline for this study. The results, shown in figure 10, agreed very closely with the closed-form solution calculated by applying equation (27). The critical moment predicted by the finite element analysis was 32,800 in-lbs, which was 5.5 percent below the closed-form value, and the cross-sectional flattening of 0.126 inches was 49 percent less than the closed-form prediction, as shown in figure 10.

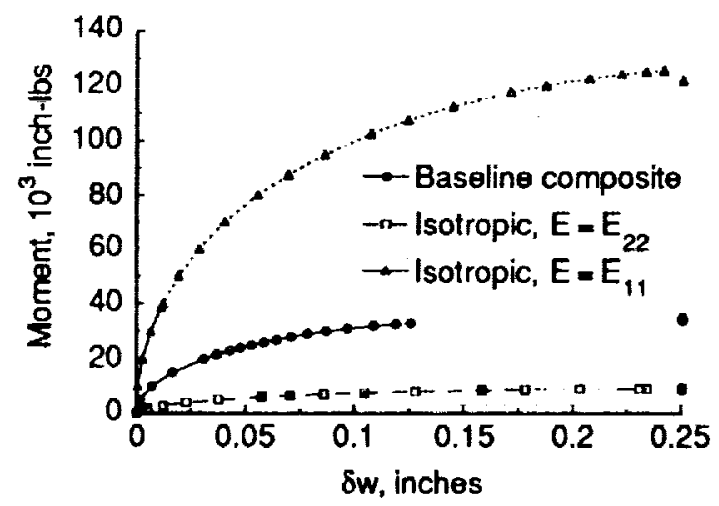

Notes:

1. Filled symbols represent predicted (closed-form) results

2. Dashed line for upper curve represents results obtained by assuming linear elastic material

Figure 10 Comparison of composite and isotropic results

Figure 11 shows a plot of the deformed tube at the point of collapse. Inspection of the deformations shows a visible difference between the top and bottom deflections, $\delta_{1}$ and $\delta_{2}$, as in the isotropic case.

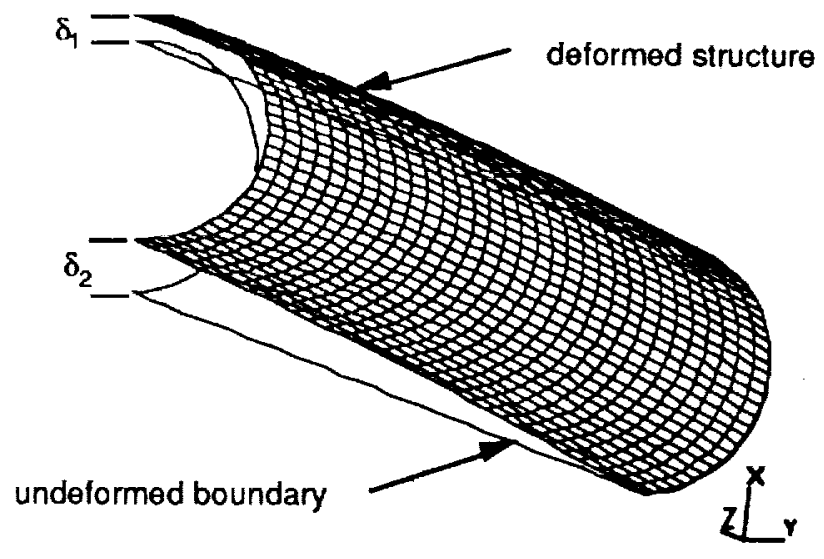

Note: Displacements are not magnified

Figure 11 Deformed structure prior to collapse, baseline composite

Figure 12 shows this effect more clearly in a composite plot of the tube cross-section for various load levels. It is evident that the bottom of the tube, which was in longitudinal compression, deformed (flattened) more than the top of the tube, which was in longitudinal tension, i.e., the tube cross-section did not deform into the doubly symmetric shape assumed in the closed-form development. This reduced deformation at the top of the tube was evidently caused by the stiffening effects of the tension field.

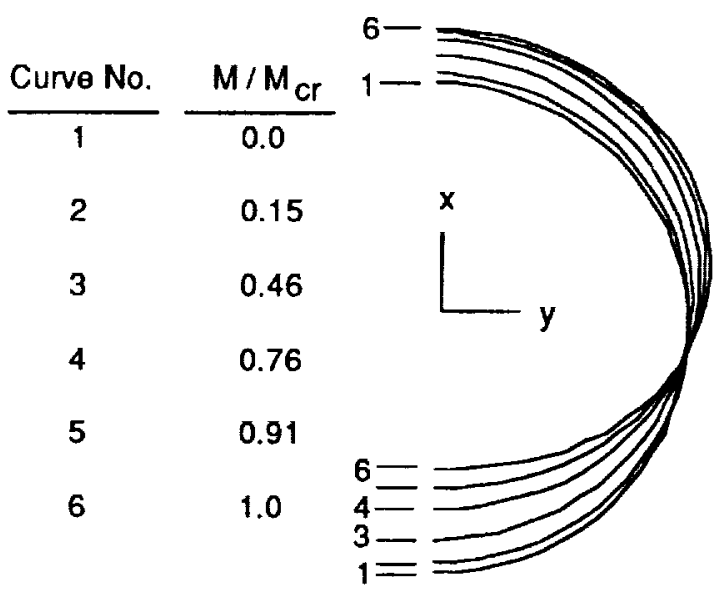

Note: displacements are not magnified

Figure 12 Cross-sectional flattening at symmetry boundary, baseline composite

The stress distribution of the finite element solution was investigated to evaluate the assumptions used in the development of the closedform solution. The longitudinal stress field shows evidence of pure bending at the center of the tube when it is near failure as was assumed in the closedform solution. In addition the circumterential stress at the middle surface of the tube, with the exception of a local stress concentration in the compression field, was very small, validating the assumption of zero circumferential strain in the closed-form solution. 


\section{Isotropic Solution as Uoper Bound}

A second isotropic case was analyzed using the longitudinal modulus, $E_{1 t}$, of the composite. The value of $E_{1}$ (17.5E6 psi) was approximately the same as Young's modulus for titanium. This case provided an upper bound for the collapse moment of the composite tube. The three solutions are plotted in figure 10. Note that the isotropic tube with $E=E_{11}$ is so much stiffer than the other two tubes, that it would have experienced material failure at a load level of about 30 percent of the predicted collapse moment. The results plotted in figure 10 show that using Reissner's formula for isotropic tubes with the weaker olastic modulus, $E_{22}$, would result in a substantially conservative prediction of the collapse moment of the composite tube.

\section{Other Possible Failure Mechanisms}

Alternative failure mechanisms were investigated to determine whether the composite tube might fail at a load level below the calculated critical moment. The closed-form predicted applied stress state was used to calculate stresses in the oliter plies of the laminate, and the results showed that laminate failure would probably not occur before the tube collapsed due to the nonlinear bending effect.

The other failure mechanism which was a concern for thin tubes was short wave-length buckling on the compression side of the tube. The formulas of reference 9 were used to calculate the critical bending moment which would cause buckling of an orthotropic cylinder. The results indicate that the minimum bending moment which would cause short-wave buckling of the tube is 42,100 in-lb. This value is greater than the predicted collapse moment of 34,700 in-lb, indicating that the Brazier effect would probably be the dominant lailure mechanism for the baseline tube.

\section{Practical Bange of Application for Tubes}

Several tubes having the same lamina properties, but different layups and thicknesses, were analyzed to provide further verification of the accuracy of the closed-form solution for a wider range of parameters. The equation for the critical moment, equation (27), is rewritten below in terms of a parameter $\lambda$ :

$$
\mathbf{M}_{\mathbf{c r}}=\boldsymbol{\pi r} \lambda
$$

where $r$, the tube radius, is a geometrical parameter, and $\lambda=\left[\mathrm{A}_{11} \mathrm{D}_{22}\right]^{1 / 2}$ is a material parameter which incorporates the thickness $h$. The parameter $\lambda$ represents an "equivalent stiffness" which accounts for the longitudinal extensional stiffness and the circumferential bending stiffness of the shell. The calculated critical bending moments for three different radii $r$ are plotted as a function of $\lambda$ in figure 13. The closed-form and finite element results shown in the ligure agree closely, with the maximum difference no greater than 7 percent. The $r / t$ ratios varied from 12 to 26 . In general, the tubes with a higher $r / t$ ratio showed closer agreement between the closed-form and finite element results. As in the baseline case, the predicted local instability due to short-wave buckling would occur at a moment greater than the collapse moment for the finite element models used in this study.

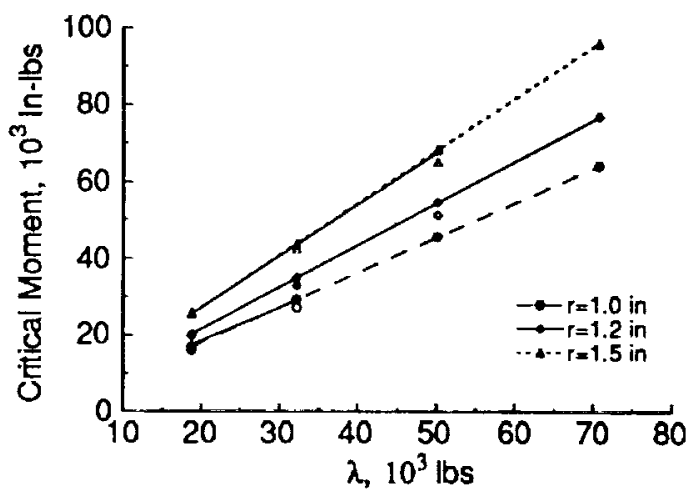

Notes: 1. Dashed lines indicate regions where material failure would probably occur at a moment less than $\mathrm{Mcr}$

2. Open symbols indicate finite element results

3. $\mathrm{M}_{\mathrm{Cr}}=\pi \mathrm{r} \lambda$ where $\lambda=\left[\mathrm{A}_{11} \mathrm{D}_{22}\right]^{1 / 2}$

Figure 13 Predicted values of $M_{c r}$ vs $\lambda$ for various composite tubes

The closed-form predicted values of stress at $M_{c r}$ were calculated for each case to determine if material failure would occur for a moment less than $M_{C r}$. The dashed-line portions of the curves in figure 13 show the values of $\lambda$ for which material failure would govern, based on strength values given in table 1. The curves should provide accurate values of $M_{c r}$ for any composite which behaves as specially orthotropic and has values of $\lambda$ in the range shown on the plot, however, the point at which the dashed lines begin would vary depending on the strength properties of the laminate. The values of $\lambda$ chosen for analysis were based on plausible symmetric layups.

\section{Conclusions}

Reissner's theory for the nonlinear collapse of isotropic circular cylinders has been extended to include composite tubes having specially orthotropic material properties subject to end moments, and a single closed-form formula has been presented as a design aid. Nonlinear finite element analyses were conducted to investigate the accuracy of the extended solution. Agreement between the closedform solution and the finite element analysis was good for the structural parameter range investigated. The finite element analysis verified the following major assumptions of the closed-form solution: (1) the cross-sectional deformation was inextensible, and (2) the longitudinal stress distribution was 
constant along the length of the tube. The finite element analysis also validated the extension of the closed-form solution proposed in this paper for specially orthotropic materials, and verified that neglect of the $D_{16}$ and $D_{26}$ terms in the bending stiffness matrix did not significantly affect the accuracy of the closed-form solution. The solution was used to investigate possible failure due to pure bending of graphite-epoxy truss members of a size consistent with tubes designed for large on-orbit space structures. Several additional nonlinear finite element analyses validated the closed-form solution for an extended range of tube thicknesses and diameters.

\section{References}

1. Reissner, E., "On Finite Bending of Pressurized Tubes," Journal of Applied Mechanics, vol. 26, Trans. ASME, September 1959, pp. 386-392.

2. Brazier, L.G., "On the Flexure of Thin Circular Shells and Other "Thin' Sections," Proceedings of the Royal Society, series A, vol. 116, 1927, pp. 104114.

3. Wood, J.D., "The Flexure of a Uniformly Pressurized, Circular, Cylindrical Shell," Journal of Applied Mechanics, vol. 25, Trans. ASME, vol. 80, 1958. pp. 104-114.

4. Axelrad, E.L. and Emmerling, F.A., "Collapse Load of Elastic Tubes Under Bending," Israel Journal of Technology, Vol. 22, 1984/5, pp. 89-94.

5. Halpin, J.C., Primer on Composite Materials: Analysis, TECHNOMIC Publishing Co., Inc., 1984.

6. Stephens, W.B., Starnes, J.H., Jr., and Almroth, B.O., "Collapse of Long Cylindrical shells under Combined Bending and Pressure Loads," AIAA Journal, Vol. 13, No. 1, 1975, pp. 20-25.

7. Knight, N.F., Jr., Macy, S.C., and McCleary, S.L., "Assessment of Structural Analysis Technology for Elastic Shell Collapse Problems," Proceedings of The Winter Annual Meeting of the American Society of Mechanical Engineers, San Francisco, California, December 10-15, 1989.

8. MSC/NASTRAN User's Manual, The MacNealSchwendler Corporation, 1989.

9. "Buckling of Thin-Walled Circular Cylinders," NASA SP-8007, National Aeronautics and Space Administration, August 1968. 


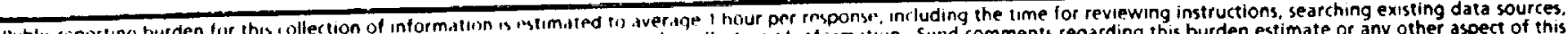

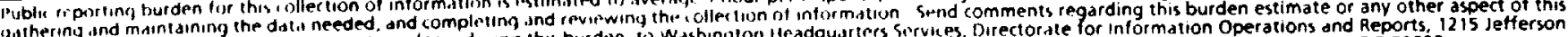

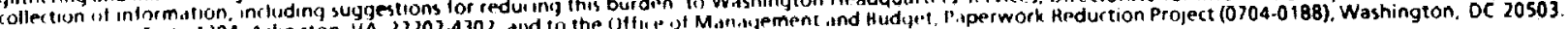

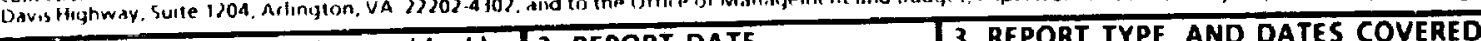

\begin{tabular}{|l|l|l}
\hline 1. AGENCY USE ONLY (Leave blank) & $\begin{array}{l}\text { 2. REPORT DATE } \\
\text { April } 1992\end{array}$ & $\begin{array}{c}\text { 3. REPORT TYPE AND DATES COV } \\
\text { Technical Memorandum }\end{array}$
\end{tabular}

\section{TITLE AND SUBTITLE}

Collapse of Composite Tubes Under End Moments

\section{FUNDING NUMBERS}

WU 590-14-31-01

\section{AUTHOR(S)}

Alan E. Stockwell and Paul A. Cooper

\section{PERFORMING ORGANIZATION NAME(S) ANO ADDRESS(ES)}

8. PERFORMING ORGANIZATION

NASA Langley Research Center

REPORT NUMBER

Hampton, VA 23665-5225

9. SPONSORING/MONITORING AGENCY NAME(S) AND ADDRESS(ES)

National Aeronautics and Space Administration

Washington, DC 20546-0001
10. SPONSORING/MONITORING AGENCY REPORT NUMBER

NASA TM- 107605

\section{SUPPLEMENTARY NOTES}

Alan E. Stockwe11: Lockheed Engineering \& Science Co., Hampton, VA; Paul A. Cooper: NASA Langley Research Center, Hampton, VA; Presented at the 33rd Structures, Structural Dynamics, and Materials Conference, April 13-17, 1992, Dallas, TX

Unclassified--Unlimited

Subject Category 39

\section{ABSTRACT (Maximum 200 words)}

Cylindrical tubes of moderate wall thickness such as those proposed for the original space station truss, may fail due to the gradual collapse of the tube cross-section as it distorts under load. Sometimes referred to as the Brazier instability, it is a nonlinear phenomenon. This paper presents an extension of an approximate closedform solution of the collapse of isotropic tubes subject to end moments developed by Reissner in 1959 to include specially orthotropic material. The closed-form solution was verified by an extensive nonlinear finite element analysis of the collapse of long tubes under applied end moments for radius-to-thickness ratios and composite layups in the range proposed for recent space station truss framework designs. The finite element analysis validated the assumption of inextensional deformation of the cylindrical cross-section and the approximation of the material as specially orthotropic.

\section{SUBJECT TERMS}

Nonlinear, Collapse, Composite, Finite Element, Tube

5. NUMBER OF PAGES

11

16. PRICE CODE

$\mathrm{AO} 3$

\begin{tabular}{|l|l|}
\hline $\begin{array}{l}\text { 17. SECURITY CLASSIFICATION } \\
\text { OF REPORT } \\
\text { Unclassified }\end{array}$ & $\begin{array}{l}\text { 18. SECURITY CLASSIFICATION } \\
\text { OF THIS PAGE } \\
\text { Unclassified }\end{array}$ \\
\hline
\end{tabular}

19. SECURITY CLASSIFICATION OF ABSTRACT
20. LIMITATION OF ABSTRACT 
